\title{
Bone Health Among Premenopausal Female Alcoholics: A Pilot Study
}

\author{
M.A. Clynes ${ }^{1}$, P. Wyawahare ${ }^{2}$, G. Robinson ${ }^{3}$, H.J. Denison ${ }^{2}$, G. Evans ${ }^{3}$, M. Gilmour ${ }^{3}$ and \\ E.M. Dennison ${ }^{1,2 *}$ \\ ${ }^{I}$ MRC Lifecourse Epidemiology Unit, Southampton, UK \\ ${ }^{2}$ Victoria University, Wellington, New Zealand \\ ${ }^{3}$ Capital and Coast District Health Board, Wellington, New Zealand
}

\begin{abstract}
We report a pilot study of bone health of alcohol dependent women.
Women admitted to an alcohol-withdrawal unit (cases) and a convenience sample of controls (nursing staff) were recruited and asked to complete a lifestyle questionnaire before undergoing heel ultrasound measurements. Fasting blood samples were obtained on the day of admission (day 1) and at 5 days. Bone turnover markers (P1NP and CTX) and vitamin D levels were measured in a subset of the alcohol dependent population.

Cases were less physically active than controls. Alcoholic women had lower heel ultrasound derived Stiffness Index scores [mean $85.2(17.6)$ ] compared with controls [mean $95.5(18.7)$ ] $(\mathrm{p}=0.07)$. P1NP rose significantly over the detoxification programme [day 1: $28.35 \mu \mathrm{g} / \mathrm{l}$ (12.25); day 5: $34.19 \mu \mathrm{g} / \mathrm{l}$ (13.16), $\mathrm{p}=0.003$ ] but CTX change was not significant.
\end{abstract}

Lifestyle factors associated with poor bone health are prevalent in female alcoholics. Significant increase in bone formation was observed 5 days after alcohol withdrawal.

Keywords: Alcohol, bone turnover markers, female, fractures, heel ultrasound, premenopausal.

\section{INTRODUCTION}

Excess alcohol consumption is known to be detrimental to bone health. In addition to a direct toxic effect of alcohol on osteoblast function, alcoholism is typically associated with other lifestyle choices that are associated with reduced bone density, such as cigarette smoking, low dietary calcium intake, and physical inactivity [1-3]. Osteoporosis (OP), which is defined as an absolute decrease in total bone mass, is therefore a commonly encountered comorbidity of alcoholism, and represents a significant public health burden as reduced bone mass significantly increases the risk of fragility fracture. At the cellular level, OP is caused by an imbalance between osteoclastic bone resorption and osteoblast-mediated bone formation, and each of these processes can be measured indirectly through markers of biochemical bone turnover (BTM).

The majority of studies demonstrating the adverse effects of alcohol on bone health have been conducted in males [3]. Although in women postmenopausal oestrogen deficiency is widely regarded as the most eminent factor leading to OP, female alcoholism is rising worldwide, particularly in young women $[4,5]$. Thus far, epidemiological data relating to bone health in female alcoholics has been lacking [6]. Here

*Address correspondence to these authors at the MRC Lifecourse Epidemiology Unit, Southampton, UK;

E-mail: Elaine.Dennison@vuw.ac.nz we report the results of a study of lifestyle factors and bone quality as assessed by heel ultrasound among premenopausal Caucasian alcohol dependent women admitted to a New Zealand detoxification centre. In addition, we report bone turnover markers measured on the day of admission, and 5 days after alcohol withdrawal as we hypothesized that recovery of bone turnover might occur shortly after alcohol withdrawal.

\section{METHODS}

\subsection{Recruitment}

Caucasian premenopausal women aged $18-45$ years, who all reported regular menstrual periods, admitted to the alcohol detoxification Unit at Kenepuru Hospital, Wellington, New Zealand were recruited in this study. Women were admitted voluntarily and came from the lower half of the North Island of New Zealand. Women were approached on the day of admission. Those women able to give informed written consent completed a lifestyle questionnaire detailing: smoking and alcohol consumption; medical history and medication use; falls and fracture history; and dietary calcium intake as a validated food frequency questionnaire [7]. Physical activity was reported using the New Zealand physical activity questionnaire [8]. A convenience sample of controls was recruited from nursing staff from the same hospital through a poster recruitment campaign. 
Table 1. Summary statistics.

\begin{tabular}{|c|c|c|}
\hline & Alcohol Dependence Group & Healthy Volunteers \\
\hline \hline Age mean (SD) & $41.7(8.04)$ & $42.3(12.95)$ \\
\hline Median daily calcium intake (range) & $411.5(35-2839) \mathrm{mg}(101-1166.3) \mathrm{mg}$ \\
\hline Current smoker & $70.6 \%$ & $6 \%$ \\
\hline Low physical activity & $35.3 \%$ & $13.3 \%$ \\
\hline Moderate physical activity & $20.6 \%$ & $80 \%$ \\
\hline Vigorous physical activity & $44.1 \%$ & $1.8(2.74)$ \\
\hline Alcohol units/day (SD) & $12.4(7.04)$ & $25 \%$ \\
\hline Falls in past year & $76.5 \%$ & $20 \%$ \\
\hline Fracture in adulthood & $44.1 \%$ & $25 \%$ \\
\hline
\end{tabular}

\subsection{Heel Ultrasound}

We elected to perform heel ultrasound measurements in this study as there was no inpatient access to dual energy x-ray absorptiometry at the unit where this study was performed, and there were significant concerns about non-attendance for scans scheduled shortly after discharge from the unit. Hence heel ultrasound was performed bilaterally using a GE Achilles+ heel ultrasound machine. Results were displayed as Broadband Ultrasound Attenuation (BUA) and Speed of Sound (SOS). Furthermore, the device combines the values of the BUA and SOS to calculate a parameter known as the Calcaneal Stiffness Index, a derivation that is considered to best equate with bone mineral density as assessed by dual energy x-ray absorptiometry (DXA), and which is calculated as stiffness $=0.28 * \mathrm{SOS}+0.67 * \mathrm{BUA}-420$. Quality Assurance (QA) was performed each day using a phantom. An average of the two heels was used for all analyses.

\subsection{Measurement of Bone Turnover Markers and Serum Vitamin D Concentration}

Blood samples were taken in a random subset of women on the day of admission, and on discharge 5 days later. These included $25(\mathrm{OH}) \mathrm{D}$, serum procollagen type $1 \mathrm{~N}$ propeptide (P1NP) and serum C-terminal cross-linking telopeptide of type 1 collagen (CTX). CTX was isolated from blood using the Roche Elecsys Beta-Crosslaps method. P1NP was isolated from blood using the Roche Elecsys 2010 method. Vitamin D was separated from blood using a Radioimmunoassay (RIA).

\subsection{Ethical Approval}

Ethics approval was sought and gained from Central Health and Disability Ethics Committee, New Zealand.

\subsection{Statistical Analysis}

Study participants' characteristics were summarised using means and standard deviations (SD) for continuous variables and numbers and percentages for binary and categorical variables. Differences in the heel ultrasound readouts between the control group and the case group were assessed using an unpaired student's T-test. Differences in the levels of bone turnover markers in the alcoholic women between the day of admission to the alcohol withdrawal unit and after 5 days of detoxification were assessed using a paired student's t test. A P value was deemed significant if it were $\leq 0.05$.

\section{RESULTS}

\subsection{Summary Statistics}

Table 1 shows the summary characteristics of the alcohol dependent group (cases) and the healthy volunteers (controls). The study population comprised 34 cases and 15 controls, of mean age 41.7 (8.04) years and 42.3 (12.95) years respectively. Cases reported alcohol consumption of 12.4 (7.04) alcohol units daily, versus 1.8 (2.74) units in controls. A high proportion of alcoholic women were current smokers $(70.6 \%)$, compared with only $6 \%$ of controls. The mean daily dietary calcium intake in cases ranged from $35 \mathrm{mg}$ to $2839 \mathrm{mg}$ daily, compared with $101 \mathrm{mg}$ to $1166.3 \mathrm{mg}$ in controls. In the case group $44.1 \%$ of the women reported vigorous levels of physical activity compared with $80 \%$ of the control group. Falls in the last year were higher in the case group than the control group $(76.5 \%$ and $25 \%$ respectively). Significantly, $44.1 \%$ alcoholics reported adulthood fractures compared with $20 \%$ in the control group.

\subsection{Heel Ultrasound Results}

Table 2 shows a comparison of heel ultrasound scan results in the alcohol dependent women and healthy volunteers. Alcoholic women had lower heel ultrasound scores for BUA, SOS and SI although these results were of borderline significance in this small study.

\subsection{Bone Turnover Markers}

Serum procollagen type $1 \mathrm{~N}$ propeptide (P1NP), a sensitive marker of bone turnover, rose significantly over the detoxification programme [day 1: $28.35 \mu \mathrm{g} / 1$ (12.25); day 5: 
Table 2. Measures of bone quality in alcohol dependent women and healthy controls.

\begin{tabular}{|c|c|c|c|c|c|}
\hline & \multicolumn{2}{|c|}{ Alcohol Dependent Women } & \multicolumn{2}{c|}{ Healthy Controls } & SD \\
\hline & Mean & SD & Mean & 27.3 & 0.09 \\
\hline \hline BUA & 107.8 & 19.0 & 121.2 & 44.5 & 0.15 \\
\hline SOS & 1551.3 & 36.5 & 1570.6 & 18.7 & 0.07 \\
\hline SI & 85.2 & 17.6 & 95.5 & \\
\hline
\end{tabular}

34.19 $\mu \mathrm{g} / \mathrm{l}$ (13.16), $\mathrm{p}=0.003$ ]. Serum C-terminal crosslinking telopeptide of type 1 collagen (CTX) levels were also measured and there was no significant change over the detoxification period in this population [day 1: 0.24 (0.17); day $50.21(0.13), \mathrm{p}=0.46]$

\subsection{Vitamin D Levels}

Circulating vitamin D levels were available in 15 of the alcohol dependent women. In this population, $6(37.5 \%)$ had a circulating vitamin D level falling below the lower limit of normal (50nmol/1). Nine women had vitamin D levels between $50-100 \mathrm{nmol} / 1(56.3 \%)$ with just one woman having a circulating vitamin D level of above $100 \mathrm{nmol} / \mathrm{l}(6.25 \%)$.

\section{DISCUSSION}

\subsection{Study Findings}

Our pilot study has demonstrated that lifestyle factors associated with poor bone health, are prevalent in female alcoholics in New Zealand. Falls and fractures are common in alcoholic women and heel ultrasound measurements showed significantly lower bone density compared to the healthy volunteers. Alcoholic women were more likely to be current smokers $(70.6 \%$ of case group compared to $6 \%$ of control group). Previous studies have shown that smoking is a risk factor for the development of OP [9]. Additionally, we have shown that dietary calcium intake was often low (but extremely variable) in this group; one alcoholic woman was receiving a daily calcium intake as low as $35 \mathrm{mg}$. Although there is no consensus on the appropriate daily calcium dose different organisations recommend between $700 \mathrm{mg}$ in the UK and 1300mg in Australia and New Zealand [10]. Inadequate dietary calcium intake results in secondary hyperparathyroidism, which in turn increases bone turnover and accelerates bone loss. Interestingly, the healthy volunteers also had a lower than expected dietary calcium intake (median $421.9 \mathrm{mg}$ ) and a higher than expected fracture rate $(20 \%)$. A study by Rafferty et al., (2011) showed that patients often prefer to increase their calcium intake through diet rather than calcium supplements and therefore recommending foods high in calcium may be an effective health intervention for both alcoholic dependent women and the wider population [11].

Vitamin D levels were measured in a subset of the alcoholic women and it was found that $37.5 \%$ could be classified as vitamin D insufficient with levels below $50 \mathrm{nmol} / \mathrm{l}$. This is likely to have a negative impact on bone health as recent evidence has indicated that both gastrointestinal calcium absorption and bone mineralisation are impeded in vitamin D insufficiency. Furthermore, vitamin $\mathrm{D}$ has been shown to be protective against falls, thus reducing fracture risk further [10]. Our observations are supported by a number of previous studies which have demonstrated an association between chronic alcoholism and low circulating levels of vitamin $\mathrm{D}[3,12]$. Our findings therefore show that in alcoholic women increasing dietary vitamin D levels, in addition to calcium, is an important potential health intervention to reduce fracture risk. This could be achieved through advising the intake of vitamin Dfortified milk and other dairy products.

We observed, using heel ultrasound, that the alcoholic women had poorer heel ultrasound scores compared with the healthy control subjects. Given the limitations of our control population, we welcomed publication of reference range ultrasound results for Australian premenopausal women [13] that reported mean BUA of $121+/ 115.2 \mathrm{~dB} / \mathrm{MHz}$, mean SOS of $1592.5+/-35 \mathrm{~m} / \mathrm{s}$ and SI of $106.3+/-17.7$ in women aged 20-29 years using the same machine. We found reduced SI scores in both our cases and controls, which may reflect the older age of our study participants. Our findings are consistent with a previous study, which investigated 834 Caucasian women undergoing detoxification and found a significantly lower bone mineral density (BMD) at the femoral neck and lumbar spine of alcoholic women compared with healthy controls [14] and contrast with previous studies which have shown no effect of alcohol intake on female BMD [3, 15]. A possible explanation as to why the aforementioned studies found no link between alcohol abuse and a diminished BMD is differing levels of circulating oestradiol between the cohorts. In the studies which found no link between alcohol abuse and BMD the age of the participants ranged from 24 to 50 years. As younger participants were included in these studies it is conceivable that the average oestradiol levels were higher, which may have served as a protective factor (Malik 2009). Indeed, in the study by Malik and colleagues all 20 of the female participants showed elevated oestradiol levels [3]. Differences in other heel ultrasound results (BUA; SOS; SI) were of borderline significance but the trend indicates a reduction in bone quality in the alcohol dependent women which we predict would become statistically significant in a larger cohort. 
While some studies have suggested that quantitative ultrasound (QUS) correlates relatively poorly in vivo with axial bone densitometry by DX [16], site-specific correlations are better [17] and, in vitro, calcaneal BUA is highly correlated with bone mineral content [18]. Further it is clear that while QUS parameters are related to bone mineral content (BMC) or BMD they also reflect other structural or mechanical bone properties including stiffness and elasticity, all of which contribute to bone strength [19]. Finally, as the World Health Organisation (WHO) has defined osteoporosis in terms of BMC or BMD in women, a diagnosis of the condition can only be based on technology which assesses these parameters alone - although of course ultrasound is likely to offer information on other aspects of bone quality [20].

Our BTM findings suggest that ethanol-induced bone damage is principally dependent on negative effects of bone formation. We observed that a marker for bone turnover (P1NP) rose significantly over the detoxification programme (day 1: $28.35 \mu \mathrm{g} / \mathrm{l}(12.25)$; day $5: 34.19 \mu \mathrm{g} / \mathrm{l}(13.16), \mathrm{p}=0.003)$ whereas a marker for bone resorption (CTX), was nonsignificant. This is consistent with previous studies. For example, in a study of alcoholic males by Kim et al. (2003), OC, an alternative marker for bone formation, was slightly decreased in the alcohol dependent group, whereas D-Pyr (a marker of bone resorption) showed no significant change [21]. Additionally, a study by Turner et al. (2000) showed that alcoholics had significantly higher bone-specific alkaline phosphatase, and lower OC than controls [22]. The significant change we can detect in bone turnover after just 5 days of abstinence is a potentially very powerful message for health promotion. Explaining to an individual who is dependent on alcohol that we can detect a positive change in their bone health after a short time of refraining from alcohol is potentially very motivating. The marker levels we observed in our group of alcohol dependent women were higher than is typically seen in this group, with typically reported reference mean circulating concentrations of 23.1 for PINP and 0.14 for CTX in premenopausal women [23].

\subsection{Limitations}

There are a number of limitations to this study. Specifically our study was small (34 cases and 15 controls), limiting our power to detect statistically significant relationships. Power calculations suggest that our sample size afforded power of $53 \%$ to detect the differences observed between cases and controls. Furthermore, the results may not be entirely representative of the New Zealand population in both the case and control groups. The case group represent a sub-section of alcohol dependent women who have chosen to attend the detoxification unit for help with their alcohol addiction and were drawn from a large geographic area in New Zealand. It is therefore reasonable to assume that these women may have a greater interest in improving their health-related behaviours than the alcoholic women who do not seek help. Equally, the control group may be subject to selection bias, as they were all recruited from nursing staff at the hospital. It is of note that the control group had a high prevalence of lifetime fractures
(20\%). A possible explanation for this is that those individuals who offered to participate in this study had fractured in the past and had therefore developed an interest in their own bone health. We used heel ultrasound for pragmatic reasons as there was no on site access to DXA (the traditional gold standard). However, heel ultrasound captures other aspects of bone quality and has been validated in premenopausal women [24]. There are also some limitations associated with the use of bone turnover markers to predict bone formation and resorption. It is recognised that several factors influence bone turnover markers, including menopausal status, sex, age, gender, food intake [25]. Finally, we used a self report of fracture and were unable to validate events through medical records.

\section{CONCLUSION}

In this small study, we have demonstrated that lifestyle factors for OP other than alcohol excess are common in female alcoholics, specifically: cigarette smoking; low levels of physical activity; low vitamin D levels; and very low dietary calcium intakes in many. We have also shown that falls and fractures are common in alcohol-dependent women and that an increase in bone turnover is detectable after just 5 days of abstinence. Further study is now warranted in larger numbers, and for longer durations to observe bone turnover recovery in this group.

\section{CONFLICT OF INTEREST}

The authors confirm that this article content has no conflict of interest.

\section{ACKNOWLEDGEMENTS}

Declared none.

\section{REFERENCES}

[1] Leslie WD, Bernstein CN, Leboff MS. AGA technical review on osteoporosis in hepatic disorders. Gastroenterology 2003; 125(3): 941-66.

[2] Gonzalez-Reimers E, Alvisa-Negrin J, Santolaria-Fernandez F, et al. Vitamin D and nutritional status are related to bone fractures in alcoholics. Alcohol Alcohol 2011; 46(2): 148-55.

[3] Malik P, Gasser RW, Kemmler G, et al. Low bone mineral density and impaired bone metabolism in young alcoholic patients without liver cirrhosis: a cross-sectional study. Alcohol Clin Exp Res 2009; 33(2): 375-81.

[4] Grant BF, Dawson DA, Stinson FS, Chou SP, Dufour MC, Pickering RP. The 12-month prevalence and trends in DSM-IV alcohol abuse and dependence: United States, 1991-1992 and 20012002. Drug Alcohol Depend 2004; 74(3): 223-34.

[5] Huerta MC, Borgonovi F. Education, alcohol use and abuse among young adults in Britain. Soc Sci Med 2010; 71(1): 143-51.

[6] Mikosch P. Alcohol and bone. Wien Med Wochenschr 2014; 164(1-2): 15-24.

[7] Montomoli M, Gonnelli S, Giacchi M, et al. Validation of a food frequency questionnaire for nutritional calcium intake assessment in Italian women. Eur J Clin Nutr 2002; 56(1): 21-30.

[8] Maddison R, Ni Mhurchu C, Jiang Y, et al. International Physical Activity Questionnaire (IPAQ) and New Zealand Physical Activity Questionnaire (NZPAQ): a doubly labelled water validation. Int J Behav Nutr Phys Act 2007; 4: 62. 
[9] Christianson MS, Shen W. Osteoporosis prevention and management: nonpharmacologic and lifestyle options. Clin Obstet Gynecol 2013; 56(4): 703-10.

[10] Levis S, Lagari VS. The role of diet in osteoporosis prevention and management. Curr Osteoporos Rep 2012; 10(4): 296-302.

[11] Rafferty K, Watson P, Lappe JM. The selection and prevalence of natural and fortified calcium food sources in the diets of adolescent girls. J Nutr Educ Behav 2011; 43(2): 96-102.

[12] Mobarhan SA, Russell RM, Recker RR, Posner DB, Iber FL, Miller P. Metabolic bone disease in alcoholic cirrhosis: a comparison of the effect of vitamin D2, 25-hydroxyvitamin D, or supportive treatment. Hepatology 1984; 4(2): 266-73.

[13] Gould H, Brennan SL, Nicholson GC, Kotowicz MA, Henry MJ, Pasco JA. Calcaneal ultrasound reference ranges for Australian men and women: the Geelong osteoporosis study. Osteoporos Int 2013; 24(4): 1369-77.

[14] Clark MK, Sowers MF, Dekordi F, Nichols S. Bone mineral density and fractures among alcohol-dependent women in treatment and in recovery. Osteoporos Int 2003; 14(5): 396-403.

[15] Laitinen K, Karkkainen M, Lalla M, et al. Is alcohol an osteoporosis-inducing agent for young and middle-aged women? Metabolism 1993; 42(7): 875-81.

[16] Grampp S, Genant HK, Mathur A, et al. Comparisons of noninvasive bone mineral measurements in assessing age-related loss, fracture discrimination, and diagnostic classification. J Bone Miner Res 1997; 12(5): 697-711.

[17] Langton CM, Langton DK. Comparison of bone mineral density and quantitative ultrasound of the calcaneus: site-matched correlation and discrimination of axial BMD status. Brit J Radiol 2000; 73(865): 31-5.

[18] Langton CM, Njeh CF, Hodgskinson R, Currey JD. Prediction of mechanical properties of the human calcaneus by broadband ultrasonic attenuation. Bone 1996; 18(6): 495-503.

[19] Nicholson PH, Muller R, Lowet G, et al. Do quantitative ultrasound measurements reflect structure independently of density in human vertebral cancellous bone? Bone 1998; 23(5): 425-31.

[20] Bouxsein ML, Radloff SE. Quantitative ultrasound of the calcaneus reflects the mechanical properties of calcaneal trabecular bone. J Bone Miner Res 1997; 12(5): 839-46.

[21] Kim MJ, Shim MS, Kim MK, et al. Effect of chronic alcohol ingestion on bone mineral density in males without liver cirrhosis. Korean J Inter Med 2003; 18(3): 174-80.

[22] Turner RT. Skeletal response to alcohol. Alcohol Clin Exp Res 2000; 24(11): 1693-701.

[23] Vasikaran S, Eastell R, Bruyere O, et al. Markers of bone turnover for the prediction of fracture risk and monitoring of osteoporosis treatment: a need for international reference standards. Osteoporos Int 2011; 22(2): 391-420.

[24] Hawker GA, Jamal SA, Ridout R, Chase C. A clinical prediction rule to identify premenopausal women with low bone mass. Osteoporos Int 2002; 13(5): 400-6.

[25] Szulc P, Delmas PD. Biochemical markers of bone turnover: potential use in the investigation and management of postmenopausal osteoporosis. Osteoporos Int 2008; 19(12): 1683704.

(C) Clynes et al.; Licensee Bentham Open.

This is an open access article licensed under the terms of the Creative Commons Attribution Non-Commercial License (http://creativecommons.org/licenses/by$\mathrm{nc} / 3.0 /$ ), which permits unrestricted, non-commercial use, distribution and reproduction in any medium, provided the work is properly cited. 